\title{
Amorphous Solid Dispersions in Early Stage of Formulation Development: Predicting Excipient Influence on Dissolution Profiles Using DDDPlus
}

Juliet Obianuju Njoku' ${ }^{1}$, Dwaipayan Mukherjee ${ }^{2}$, Gregory K. Webster ${ }^{2}$, and Raimar Löbenberg ${ }^{1 *}$

${ }^{1}$ Faculty of Pharmacy and Pharmaceutical Sciences, Katz Group-Rexall Centre for Pharmacy and Health Research, University of Alberta, Edmonton, Canada.

${ }^{2}$ Research and Development, AbbVie, Inc., North Chicago, IL, USA.

e-mail: raimar@ualberta.ca

\section{ABSTRACT}

Excipients play an important role in the formulation of dosage forms and can be used to improve the bioavailability of a drug through physical interactions that alter the rate of dissolution of a drug. The objective of this study was to predict the effect of formulation on the dissolution rate of a poorly soluble drug using computer simulations. Solid dispersion of ritonavir was prepared. Dissolution test results of direct compressed tablets with and without disintegrant in various media with physiologically relevant $\mathrm{pH}$ were compared with simulations. Solubilizer and disintegrant effect were evaluated on the Dose, Disintegration, and Dissolution Plus (DDDPlus) simulation software (version 5.0.0011, Simulations Plus, Inc., Lancaster, CA, USA) using previously published solubility data on ritonavir. Observed and predicted dissolution profiles similarity tests and drug release mechanisms were assessed. Optimization of the solubilizer effect coefficient (SEC) on the program gives good estimations of the effect of copovidone in the solid dispersion in the dissolution profiles of all tablets. The SEC is dependent on the solubility of the active pharmaceutical ingredient (API) at the local $\mathrm{pH}$ and the dissolved concentration of the solubilizer. Disintegrant concentration in the program has no effect on simulations, rather the disintegration time was the predictive factor. Drug release was formulation controlled in the tablets without disintegrant and in the tablets with disintegrant was via drug diffusion and polymer surface erosion. DDDPlus has the potential to estimate the effect of excipients in a formulation on in vitro dissolution at an early stage in the drug development process. This could be useful in decisions on formulation strategies to enhance bioavailability in poorly soluble drugs.

KEYWORDS: Dissolution, simulations, solid dispersion, solubilizer effect coefficient

\section{INTRODUCTION}

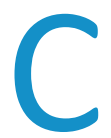

rystalline solids are more commonly used in pharmaceutical formulations due to their chemical and physical stability. The crystalline property has negative effects on a drug's solubility and dissolution, especially for Biopharmaceutics Classification System (BCS) class II and IV drugs (1). Low solubility is a notable hindrance to the effective delivery of therapeutic agents because the absorption of orally administered drugs depends on dissolution and gastrointestinal permeability (2). The use of high-energy forms such as amorphous solid dispersions (ASDs) can improve drug solubility and consequently delivery. Poorly water-soluble drugs, when in the amorphous state, have higher solubility because no energy is required to break the crystal lattice during the dissolution process (3).
Solid dispersions are systems where one component is dispersed in a carrier (usually a polymer and amorphous) and the whole system appears to be in a solid state (4). Solid dispersions have larger surface area, improved wettability, and higher porosity, all of which hasten drug release when compared to the crystalline form of an active pharmaceutical ingredient (API) $(5,6)$. Solid dispersions of poorly soluble drugs require a polymer with some hydrophilic properties capable of forming intermolecular interactions with the drug (7). The polymer, copovidone (polyvinylpyrrolidone), was used as a solubilizer in this study to disperse ritonavir API into a solid state formulation.

Ritonavir, the model drug used for this study is an HIV1 protease inhibitor that inhibits the production of the structural and functional proteins of the HIV virus $(8$,

* Corresponding author. 
9). It is poorly soluble at a high $\mathrm{pH}(0.57 \mathrm{mg} / \mathrm{mL}$ in 0.1 $\mathrm{M} \mathrm{HCl}, 0.002 \mathrm{mg} / \mathrm{mL}$ at $\mathrm{pH} 6.8$ ) and a substrate of the P-glycoprotein transporter $(10,15)$.

Dose, Disintegration and Dissolution Plus (DDDPlus) software (version 5.0.0011, Simulations Plus, Inc., Lancaster, CA, USA) is a software platform that models and simulates the in vitro dissolution of API and formulation excipients in various dosage forms under various experimental conditions (11). During drug development, in vitro dissolution testing is an important tool for evaluating candidate formulations and API interaction with excipients (11). There is an emerging trend in the industry to explore alternatives to dissolution testing and to apply them during product development to ensure product quality instead of relying on traditional dissolution testing (12). The use of DDDPlus for in silico predictions along with more traditional in vitro measurements was evaluated as part of the workshop titled "Dissolution And Translational Modeling Strategies Enabling PatientCentric Drug Product Development," held in May 2017 and attended by members from worldwide regulatory agencies and consortia involved in drug development (12). Studies of drug-excipient interaction represent an important phase in the preformulation stage of dosage forms (13). The application of in silico methods to predict drug-excipient interaction and influence on formulation dissolution has the potential to expedite preformulation studies of new drugs.

The objective of this study was to assess formulation specific models in simulating drug-excipient interaction using DDDPlus, by determining the impact of prediction factors in the program on solubilizer and disintegrant effect on the dissolution profile of an immediate release, poorly soluble drug. All in silico simulations were compared with in vitro measurements to confirm prediction accuracy. This strategy can be used in designing formulation strategies in early drug development with fewer laboratory experiments involved.

\section{MATERIALS AND METHODS}

Ritonavir was provided by AbbVie, Inc. (North Chicago, IL, USA). Microcrystalline cellulose (Avicel PH-102 NF) was purchased from FMC Corp. (Philadelphia, PA, USA). Colloidal silicone dioxide was purchased from Cabot Corporation (Tuscola, IL, USA). Copovidone (Kollidon VA64) was purchased from BASF SE (Ludwigshafen, Germany). Croscarmellose sodium was obtained from PCCA Canada (London, ON, Canada). Magnesium stearate was obtained from H.L. Blachford, Ltd (Mississauga, $\mathrm{ON}$, Canada). Hydrochloric acid $(\mathrm{HCl})$ p.a. $36.5 \%$ was obtained from Thermo Fisher Scientific (Fair Lawn, NJ, USA). High-performance liquid chromatography (HPLC)grade water was obtained from Thermo Fisher Scientific, and water for the dissolution test media was produced by reverse osmosis (ELGA LabWater, UK) and filtered through a Durapore 0.22- $\mu \mathrm{m}$ GV membrane (Millipore Canada, Ltd., Etobicoke, ON, Canada) for HPLC mobile phase. Acetonitrile used in the preparation of the HPLC mobile phase was obtained from VWR International, LLC. (Radnor, PA, USA) and filtered through a Durapore 0.45$\mu \mathrm{m}$ HV membrane (Millipore Canada, Ltd).

\section{Methods}

The solid dispersion composed of ritonavir, colloidal silicon dioxide, and copovidone at a ratio of 15:1:84 (w/w/w) was prepared by melting copovidone and colloidal silicon dioxide at $150^{\circ} \mathrm{C}$ in a beaker placed in a silicone oil bath. Ritonavir was added to the molten excipients, mixed thoroughly at same temperature, and the mixture was cooled to room temperature. The mixture was ground in a mortar to powder form and stored in a desiccator. The powdered mixture was used to prepare tablets with and without disintegrant (croscarmellose sodium) by direct compression at one metric ton pressure for 30 seconds and 1 minute respectively, using a Carver Laboratory Press (Carver, Inc., Wabash, IN, USA). The composition of each tablet type is described in detail in Table 1.

Table 1. Tablet Composition With and Without Disintegrant

\begin{tabular}{|c|c|c|c|c|}
\hline & \multicolumn{2}{|c|}{ With Disintegrant } & \multicolumn{2}{c|}{ Without Disintegrant } \\
\hline Ingredient & $\begin{array}{c}\text { Amount } \\
(\mathrm{mg})\end{array}$ & $\%$ content & $\begin{array}{c}\text { Amount } \\
\text { (mg) }\end{array}$ & $\%$ content \\
\hline Ritonavir ASD & 100.00 & 11.57 & 80.0 & 80.0 \\
\hline $\begin{array}{c}\text { Microcrystalline } \\
\text { Cellulose }\end{array}$ & 586.67 & 67.87 & 19.0 & 19.0 \\
\hline $\begin{array}{c}\text { Croscarmellose } \\
\text { Sodium }\end{array}$ & 174.89 & 20.23 & - & - \\
\hline $\begin{array}{c}\text { Magnesium } \\
\text { Stearate }\end{array}$ & 2.86 & 0.33 & - & - \\
\hline $\begin{array}{c}\text { Colloidal Silicon } \\
\text { Dioxide }\end{array}$ & - & - & 0.5 & 0.5 \\
\hline $\begin{array}{c}\text { Sodium Stearyl } \\
\text { Fumarate }\end{array}$ & - & - & 0.5 & 0.5 \\
\hline
\end{tabular}

ASD: amorphous solid dispersions.

\section{Solubility and Dissolution Testing}

The solubility of the ASD was determined via the shakeflask method. Five $\mathrm{mg}$ of the powder was added to $5 \mathrm{~mL}$ of each medium $(0.1 \mathrm{M} \mathrm{HCl}, 0.01 \mathrm{M} \mathrm{HCl}$, and phosphate buffer [pH 6.8]), the solution was placed in a shaker (Heraeus Instruments, Inc., USA) for 72 hours at $25^{\circ} \mathrm{C}$. 
Samples $(1 \mathrm{~mL})$ were withdrawn without replacement at each time point $(24,48$, and 72 hours) and centrifuged at $15,000 \mathrm{rpm}$. The supernatant $(500 \mu \mathrm{L})$ was withdrawn and transferred into $2.5-\mathrm{mL}$ vials for HPLC analysis.

The $\mathrm{pH}$ of the media was measured using an Accumet $\mathrm{XL}$ $20 \mathrm{pH}$ meter (Thermo Fisher Scientific). The media was deaerated by filtration, ultrasound, and vacuum. The dissolution testing was performed using a VK 7020 system (Agilent, Santa Clara, CA, USA) equipped with $70 \mu \mathrm{m}$ Full Flow filters (Varian, Inc.) and a VK 8000 autosampler (Varian, Inc.). Dissolution tests were performed with USP apparatus 2 and $900 \mathrm{~mL}$ of dissolution medium (0.1 M $\mathrm{HCl}, 0.01 \mathrm{M} \mathrm{HCl}$, and phosphate buffer) at $75 \mathrm{rpm}$ rotation speed. Samples $(1.0 \mathrm{~mL})$ were withdrawn in triplicate without replacement at each time point $(3,5,10,15,20$, 30,45 , and 60 minutes) for HPLC analysis.

\section{HPLC Analysis}

A previously published method was used (14). In brief, a calibration curve was prepared for a range from 3.75\% to $120 \%$ of the expected maximum drug concentrations in each medium and the correlation coefficient $\left(R^{2}\right)$ for the calibration curve was greater than or equal to 0.998 . The mobile phase (acetonitrile, water, and trifluoracetic acid at a ratio of 57:43:0.1 [v/v/v]) was deaerated before use, using both vacuum filtration and ultrasound, and the flow rate was set to $1 \mathrm{~mL} / \mathrm{min}$. For the analysis, a Class-VP Series liquid chromatograph by the Shimadzu Corporation (Kyoto, Japan) was composed of a CBM-20A system controller, two LC-10AS pumps, an SIL-10ADVP autosampler, and an SPD-M10AVP diode array detector was used. The analytical column was a LiChrospher 60 RP-select B column $(5 \mu \mathrm{m}, 12.5 \times 4 \mathrm{~mm})$ (Merck KGaA, Darmstadt, Germany). An injection volume of $50 \mu \mathrm{L}$ was used without dilution and the retention time for ritonavir was approximately 4 minutes, with a total run time of 8 minutes. A wavelength of $240 \mathrm{~nm}$ was selected for the analysis.

\section{DDDPlus Simulation}

DDDPlus is a software program that simulates the dissolution profile of a formulation through its ingredients' physicochemical properties and the dissolution test conditions. The software has three main tabs: formulation, dissolution method, and simulation $(11,14)$.

In this study, the software was used to predict the active ingredient-excipient interaction in the formulation. The formulation composition was defined by selecting all the ingredients and their functions from the included database. The immediate release (IR) tablet dosage form was selected for all simulations. The physical dimensions and manufacturing properties of the tablets consistent with the tablet compression process were entered into the software platform. Previous studies have shown that a solubility versus $\mathrm{pH}$ profile can be created by the program from a drug's experimentally determined solubility using known pKa and other physicochemical properties of the API (15). Also, one data point of measured solubility was found to be sufficient to create a solubility versus $\mathrm{pH}$ profile for simulations, therefore the solubility of ritonavir API was determined experimentally to create a solubility versus $\mathrm{pH}$ profile. The solubility of ritonavir drug powder at $\mathrm{pH} 1.0(0.57 \mathrm{mg} / \mathrm{mL})$ was used as the reference solubility (Table 2). The solubilizer constant for the solubilizer, polyvinylpyrrolidone (PVP), was calibrated to fit the concentration of solubilizer in the tablet (optimization). Optimization estimates the effect of a different concentration of the solubilizer within the formulation on the dissolution profile. This solubilizer constant empirically describes the interaction between the solubilizer and the active ingredient. The optimization module in the program was used to build the formulation-specific model. The unity objective function was employed in the optimization process with no weighting of experimental data points. Optimization was done individually for each dissolution profile in each medium. The formulation-specific model can be used to estimate the probable changes in dissolution when excipient content and experimental parameters are varied. DDDPlus models the effect of solubilizers on ingredients solubility using the equation:

$$
S_{e}=S_{A P I}(p H) \times\left[1+\sum k_{S E, i} C_{D, i}\right],
$$

where $S_{e}$ is the solubility of the solid dispersion, $S_{A P I}(p H)$ is the active drug's solubility at the local $\mathrm{pH}$ without solubilizer, $\mathrm{k}_{S E, i}$ is an optimizable coefficient for the $\mathrm{i}^{\text {th }}$ solubilizer called the Solubilizer Effect parameter (units of $\mathrm{L} / \mathrm{mg}$ ), and $C_{D, i}$ is the dissolved concentration of the $\mathrm{i}^{\text {th }}$ solubilizer.

The dissolution parameters were defined according to the following test conditions: USP apparatus 2 (paddle), $900 \mathrm{~mL}$ of medium, $75 \mathrm{rpm}$ rotation speed, and three different medium types $(0.1 \mathrm{M} \mathrm{HCl}, 0.01 \mathrm{M} \mathrm{HCl}$, and phosphate buffer [pH 6.8]). 
Table 2. Ritonavir and ASD Solubility Comparison in Different Media

\begin{tabular}{|c|c|c|}
\hline Media & $\begin{array}{c}\text { API } \\
\text { Solubility } \\
(\mathrm{mg} / \mathrm{mL})^{*}\end{array}$ & $\begin{array}{c}\text { ASD } \\
\text { Solubility } \\
(\mathrm{mg} / \mathrm{mL})\end{array}$ \\
\hline $0.1 \mathrm{M} \mathrm{HCl} \mathrm{(pH} \mathrm{1)}$ & 0.57 & 0.96 \\
\hline $0.01 \mathrm{M} \mathrm{HCl}(\mathrm{pH} 2)$ & 0.01 & 0.31 \\
\hline Phosphate buffer USP $(\mathrm{pH} \mathrm{6.8)}$ & 0.002 & 0.06 \\
\hline
\end{tabular}

* Ritonavir solubility was measured by Njoku et al, 2019

API: active pharmaceutical ingredient; ASD: amorphous solid dispersions.

Single simulations were performed for each in silico experiment using 60 minutes as the length of simulation, consistent with the experimental design. The predictions of the dynamic dissolution from DDDPlus were compared to the in vitro results.

\section{Statistical Analysis}

DDSolver an add-in for Microsoft Excel for dissolution profile data analysis, was used to evaluate and compare in vitro and in silico results. Observed and simulated dissolution profiles were compared using the statistical test for similarity $\left(f_{2}\right)$. Only percent-dissolved values less than $85 \%$ were chosen for the similarity test. For cases where most values were above $85 \%$, the lowest four values were chosen. The Korsmeyer-Peppas model in DDSolver was used to determine the drug release mechanism. Only percent-dissolved values less than $65 \%$ were chosen for the Korsmeyer-Peppas model fitting and in cases where most of the values were above $65 \%$, the first three values were used. The first-order, zero-order, Gompertz, and Hopfenberg models were also evaluated using the DDSolver.

\section{RESULTS}

Solubility tests on ritonavir ASD confirmed the $\mathrm{pH}$ dependent solubility of ritonavir as shown in Table 2. There was an improvement on the solubility of ritonavir due to excipient (solubilizer) effect.

The dissolution test results of the tablets with disintegrant are shown in Figure 1A. Predictions showed similarity to observed values in all media. There was a reduction in the fraction dose dissolved at 20 minutes in the medium of $\mathrm{pH}$ 2 and at 15 minutes in the medium of $\mathrm{pH}$ 6.8. This could be attributed to measurement variability at these time points. The $f_{2}$ value between observed and predicted profiles is shown in Table 3.

The dissolution test results of the tablets without disintegrant are shown in Figure 1B. Predictions showed a high similarity with observed values in all media used.

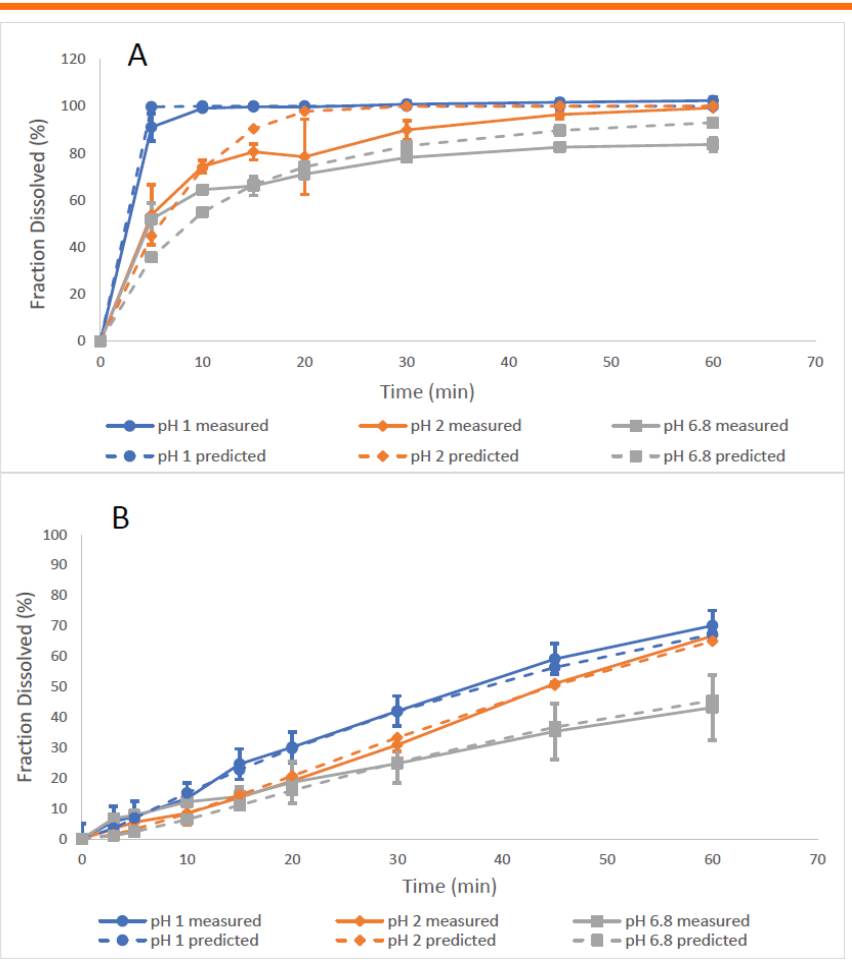

Figure 1. Dissolution of ritonavir ASD 100-mg tablets with disintegrant in different media and simulated profiles (A) and dissolution of ritonavir ASD 80-mg tablets without disintegrant in different media and simulated profiles (B). ASD: amorphous solid dispersions.

Table 3. Comparison of In Silico to In Vitro Data for Tablet Dissolution Under Various Conditions

\begin{tabular}{|c|c|c|c|c|c|}
\hline \multicolumn{2}{|c|}{ Dissolution Profile } & $f_{2}$ Value & $R^{2}$ & $n$-value & SEC \\
\hline \multirow{4}{*}{ Tablet With Disintegrant } & $\mathrm{pH} 1.0$ & 73 (yes) & 0.88 & 0.086 & 0.73 \\
\cline { 2 - 6 } & $\mathrm{pH} 2.0$ & 52 (yes) & 0.74 & 0.362 & 3.51 \\
\cline { 2 - 6 } & $\mathrm{pH} 6.8$ & 54 (yes) & 0.78 & 0.221 & 14.99 \\
\hline \multirow{3}{*}{$\begin{array}{c}\text { Tablets Without } \\
\text { Disintegrant }\end{array}$} & $\mathrm{pH} 1.0$ & 85 (yes) & 0.99 & 0.885 & 0.53 \\
\cline { 2 - 6 } & $\mathrm{pH} 2.0$ & 87 (yes) & 0.99 & 1.177 & 0.22 \\
\cline { 2 - 6 } & $\mathrm{pH} 6.8$ & 71 (yes) & 0.87 & 0.773 & 4.25 \\
\hline
\end{tabular}

SEC: solubilizer effect coefficient.

The Korsmeyer-Peppas model is expressed as $f_{t}=K t^{n}$, where $f_{t}$ is the fraction of the drug released at time $t$, $K$ is a release rate constant, and $n$ is the exponent of release (16). Plotting logarithms of fraction dissolved versus logarithm of time helps estimate a value of $n$, which can be used to identify mechanisms of dissolution. Analysis of the Korsmeyer-Peppas equation with the data resulted in n-values of $0.086,0.362$, and 0.221 (tablets with disintegrant) and $0.885,1.177$, and 0.773 (tablets without disintegrant) in media with $\mathrm{pH} 1, \mathrm{pH} 2$, and $\mathrm{pH}$ 6.8 , respectively (Table 3 ). This indicates that the drug release from the tablets with disintegrant (with $n$ values $<0.43$ ) was controlled by Fickian diffusion $(14,17)$. After model fitting with DDSolver, tablets with disintegrants fit 
well with the first-order model $\left(R^{2}\right.$ adj $=0.894,0.775$, and $0.701)$ and Gompertz model $\left(R^{2}\right.$ adj $=0.870,0.933$, and $0.977)$, which describe drug release from systems where the release rate is concentration dependent. These tablets also fit well with the Hopfenberg model $\left(R^{2}\right.$ adj $>$ $0.73)$, which describes drug release from surface eroding polymers (18). All of this suggests that the drug release for tablets with disintegrant was governed by Fickian diffusion due to the presence of disintegrant that increased surface area and enhanced dissolution of the tablets but drug release from the ASD particles was mainly formulation controlled. The tablets without disintegrant resulted in n-values (Korsmeyer-Peppas equation) that were higher or equal to 0.89 , which suggested a non-Fickian release mechanism. These tablets also fit well with the zero-order and Hopfenberg models $\left(R^{2}\right.$ adj $\left.>0.93\right)$, which indicates that, without disintegrant, the drug is released via surface erosion of the polymer and is therefore controlled by formulation factors.

The solubilizer effect coefficient (SEC), which estimates the interaction effect of the solubilizer (copovidone) on the dissolution of the ASD tablet, was calibrated for each dissolution condition. The results of this calibration are shown in Figure 2, where the solubilizer has a more pronounced effect for situations not conducive to dissolution of the tablet, i.e., absence of disintegrant and higher $\mathrm{pH}$. The influence of the SEC is also more variable for cases with higher $\mathrm{pH}$ due to slower dissolution.

\section{DISCUSSION}

The polymer matrix carrier in which the active API is homogenously dispersed contains excipients that are capable of controlling the drug release rate. The shear mixing of the molten mass during preparation of the ASD causes dispersion of the drug into the polymer matrix at a molecular level along with the possibility of drug-polymer interactions (19). The excipient, which is the ratecontrolling material, can be water-soluble or swellable (hydrophilic matrix) such as polyvinylpyrrolidone (used in this analysis) or water-insoluble (hydrophobic or inert matrix) (20). The rate at which a drug is released from the swellable, hydrophilic matrix is determined by processes such as hydration of the polymer that leads to swelling, diffusion of the drug through the hydrated polymer, drug dissolution, and polymer erosion (21). These processes occur simultaneously to facilitate drug release. The factors that influence drug release in hydrophilic matrices, such as solid dispersions, are the drug solubility, polymer viscosity, drug/polymer ratio, amount of water entering the matrix, and compression force (22).

Embedding the drug in a complex matrix usually delays the onset of dissolution of immediate release tablets. Disintegrants should therefore be added to the
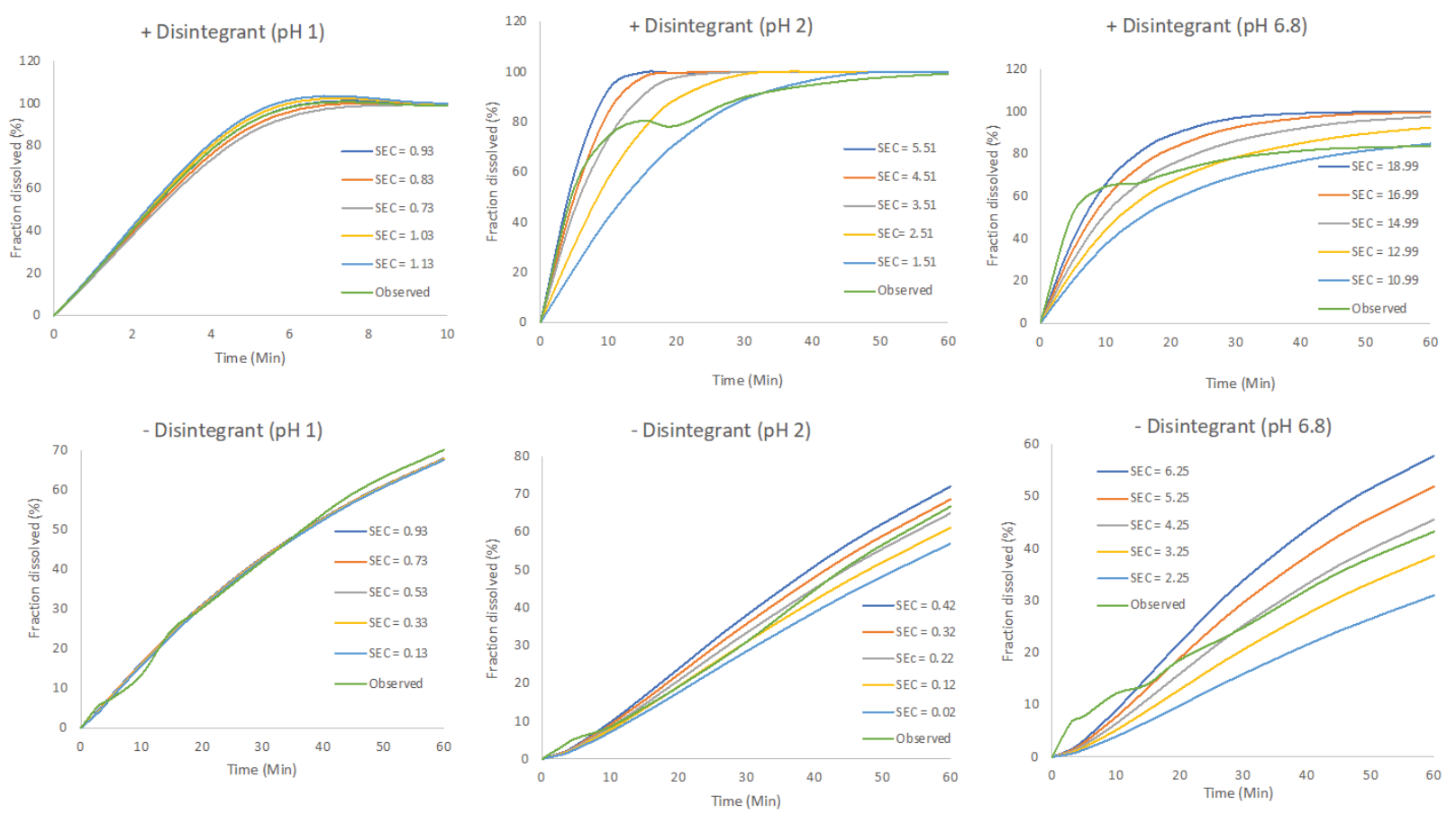

Figure 2. Comparison of observed dissolution profiles with predicted dissolution profiles with different SEC values. SEC: solubilizer effect coefficient. 
formulation to promote the breaking up of the tablet into small granules and constituent particles leading to faster liberation of the drug particles from the tablet matrix resulting in an increased surface area for subsequent dissolution (23). Copovidone has high binding and gelling properties; hence, when present in large amounts in the solid dispersion, it can result in an increased disintegration time of tablets (24). For this reason, when a high concentration of disintegrant ( $20 \%$ of croscarmellose sodium) was used in the tablets, the disintegration time was significantly decreased. The tablets without disintegrant had a prolonged disintegration time, lasting over 60 minutes. Although the dissolution process of the ASD tablets was formulation controlled, the presence of disintegrant in some of the tablets enhanced drug release, and in those tablets, dissolution was controlled by the particle properties due to fast and complete tablet disintegration (14). The suggested mechanisms of drug release for the two tablet types are summarized in Figure 3.

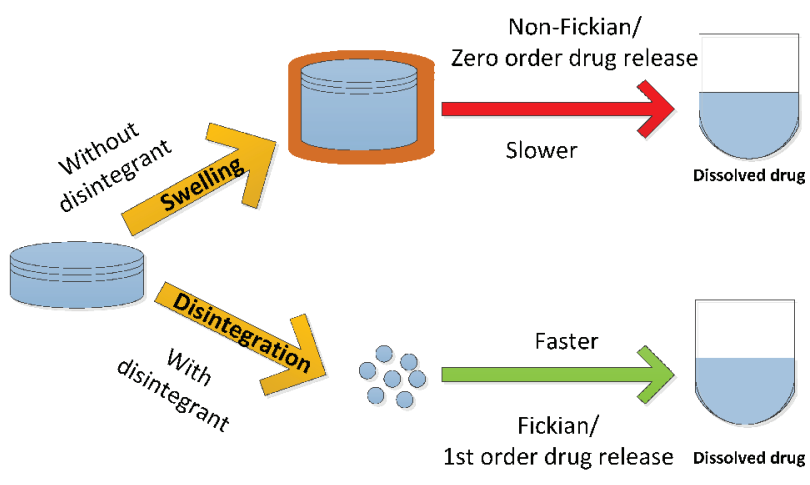

Figure 3. Probable dissolution mechanisms based on mechanistic understanding of the processes.

The Mass Transfer Model uses an empirical relationship that accounts for solubilizer effect on the dissolution rate. In this model, calibrating the solubilizer effect coefficient for one solubilizer amount will provide estimations of the effects of differing amounts of the same solubilizer on the active ingredient's dissolution. During optimization of the solubilizer effect, a change in the polymer concentration results in a different solubilizer effect parameter and, consequently, a different dissolution profile. Thesolubilizer effect parameter has an inverse relationship with the drug/polymer ratio. If the drug/polymer ratio is low, the solubilizer effect is enhanced, and a higher percentage of the drug is dissolved. Tablets with and without disintegrant had different solubilizer effect coefficients because, although the drug/polymer ratio in both tablets were the same, the overall concentration of solubilizer in the tablets was different and the tablets had varying solubility depending on the media. Also, the dissolved concentration of the solubilizer $\left(C_{D}\right)$ was influenced by the difference in disintegration time between the two tablets, which in turn was impacted by the higher polymer concentration and absence of disintegrant in the tablet without disintegrant. Optimization of the solubilizer effect for all tablets produced simulations with acceptable similarity to observed profiles, as shown by the $f_{2}$ values (Table 3). Although the amount of the disintegrant has no effect on simulations in the program if the IR tablet dosage form option is selected, the disintegration time is an important factor in estimating the rate of drug release especially in the early time points.

\section{CONCLUSION}

Simulation of dissolution profiles for immediate release and controlled release tablets involves choosing the appropriate dosage form in the program, input of disintegration time, API solubility versus $\mathrm{pH}$, and optimization of excipient effect. DDDPlus simulation software, when used with the right data, can be used in determining formulation strategies during early drug development due to its ability to predict the effect of an excipient on API solubility and dissolution rate if the excipient is identified on the software and the excipient effect is optimized. Prediction of excipient influence on the dissolution profile of a drug using DDDPlus involves quantifying the interaction between the active ingredient and the excipient. The solubility of the active ingredient in the media for dissolution has to be determined experimentally and the tablet dimensions have to be entered in the program. Other physicochemical parameters of the drug such as its molecular weight, $\mathrm{p} K_{\mathrm{a}}$, and $\log D$, which are required in DDDPlus, can be obtained from existing data that is typically present during the drug development process. The API's solubility in the dissolution medium has to be determined experimentally. The function of each excipient has to be selected in the software as the program has empirical relationships that define each function. The influence of the excipient on the active ingredient's solubility has to be defined and enhanced through optimization. It was found that a combination of these methods can achieve suitable simulations of dissolution profiles, which compared well to in vitro measurements. The use of this in silico tool, in this manner can assist in decisions concerning the choice of suitable excipients to be used in the formulation. It can 
reduce the number of laboratory experiments that are typically needed to study drug-excipient interaction and thus can shorten the overall time frame of the formulation development process.

\section{FUNDING}

This project was supported by Simulations Plus, AbbVie, and the Drug Development and Innovation Centre of the University of Alberta.

\section{CONFLICT OF INTEREST}

Juliet Obianuju Njoku is a graduate student and Raimar Löbenberg is a professor at the Faculty of Pharmacy and Pharmaceutical Sciences at the University of Alberta. Dwaipayan Mukherjee and Gregory Webster are employees of AbbVie and may hold AbbVie stocks or options. AbbVie jointly participated in the interpretation of data, writing, reviewing, and approving the publication. The presentation contains no proprietary AbbVie data.

\section{REFERENCES}

1. Newman, A.; Knipp, G.; Zografi, G. Assessing the performance of amorphous solid dispersions. J. Pharm. Sci. 2012, 101,13551377. DOI: 10.1002/jps.23031.

2. Sarode, A. L.; Wang, P.; Obara, S.; Worthen, D. R. Supersaturation, nucleation, and crystal growth during single and biphasic dissolution of amorphous solid dispersions: polymer effects and implications for oral bioavailability enhancement of poorly water soluble drugs. Eur. J. Pharm. Biopharm. 2014, 86, 351-360. DOI: 10.1016/j.ejpb.2013.10.005.

3. Taylor, L. S.; Zografi, G. Spectroscopic characterization of interactions between PVP and indomethacin in amorphous molecular dispersions. Pharm. Res. 1997, 14, 1691-1698.

4. Kolter, K.; Karl, M.; Gryczke, A. Hot-Melt Extrusion with BASF Pharma Polymers: Extrusion Compendium, $2^{\text {nd }}$ ed.; BASF SE Pharma Ingredients \& Services: Ludwigshafen, Germany, 2012.

5. Vasconcelos, T.; Sarmento, B.; Costa, P. Solid dispersions as strategy to improve oral bioavailability of poor water soluble drugs. Drug Discov. Today. 2007, 12, 1068-1075. DOI: 10.1016/j. drudis.2007.09.005.

6. Pinho, L. A. G.; Souza, S. G.; Marreto, R. N.; Barreto, L. L.; Gratieri, T.; Gelfuso, G. M.; Cunha-Filho, M. Dissolution enhancement in cocoa extract, combining hydrophilic polymers through hotmelt extrusion. Pharmaceutics. 2018; 10, 135. DOI: 10.3390/ pharmaceutics10030135.

7. Newman A. Pharmaceutical Amorphous Solid Dispersions; John Wiley \& Sons Inc: Hoboken, NJ, 2015.

8. Augustine, R.; Ashkenazi, D. L.; Arzi, R. S.; Zlobin, V.; Shofti, R.; Sosnik, A. Nanoparticle-in-microparticle oral drug delivery system of a clinically relevant darunavir/ritonavir antiretroviral combination. Acta Biomater. 2018, 1, 344-359. DOI: 10.1016/j. actbio.2018.04.045.
9. Law, D.; Krill, S. L.; Schmitt, E. A.; Fort, J. J.; Qiu, Y.; Wang. W.; Porter, W. R. Physicochemical considerations in the preparation of amorphous ritonavir-poly(ethylene glycol) 8000 solid dispersions. J. Pharm. Sci. 2001, 90, 1015-1025. DOI: 10.1002/ jps.1054.

10. NORVIR (ritonavir) Label. United States Food and Drug Administration website. https://www.accessdata.fda.gov/ drugsatfda_docs/label/2017/209512lbl.pdf. (accessed 6 Nov 2018).

11. DDDPlus User Manual. Simulations Plus Inc. 2016.

12. Abend, A.; Curran, D.; Kuiper, J.; Lu, X.; Li, H.; Hermans, A.; Kotwal, P.; Diaz, D. A.; Cohen, M. J.; Zhang, L.; Stippler, E.; Drazer, G.; Lin, Y.; Raines, K.; Yu, L.; Coutant, C. A.; Grady, H.; Krämer, J.; Pope-Miksinki, S.; Suarez-Sharp, S. Dissolution testing in drug product development: workshop summary report. AAPS J. 2019, 21, 21. DOI: 10.1208/s12248-018-0288-4.

13. Patel, B. B.; Patel, J. K.; Chakraborty, S.; Shukla, D. Revealing facts behind spray dried solid dispersion technology used for solubility enhancement. Saudi Pharm. J. 2015, 23, 352-365. DOI: 10.1016/j.jsps.2013.12.013.

14. Uebbing, L.; Klumpp, L.; Webster, G. K.; Löbenberg, R. Justification of disintegration testing beyond current FDA criteria using in vitro and in silico models. Drug Des. Devel. Ther. 2017, 11(11), 1163-1174. DOI: 10.2147/DDDT.S131213.

15. Njoku, J. O.; Amaral Silva, D.; Mukherjee, D.; Webster, G. K.; Löbenberg, R. In silico tools at early stage of pharmaceutical development: data needs and software capabilities. AAPS PharmSciTech. 2019, 20, 243. DOI: 10.1208/s12249-019-1461-5.

16. Korsmeyer, R. W.; Gurny, R.; Doelker, E.; Buri, P.; Peppas, N A. Mechanisms of solute release from porous hydrophilic polymers. Int. J. Pharm. 1983, 15, 25-35. DOI: 10.1016/03785173(83)90064-9.

17. Ritger, P. L.; Peppas, N. A. A simple equation for description of solute release I. Fickian and non-Fickian release from nonswellable devices in the form of slabs, spheres, cylinders or discs. J. Control. Release. 1987, 5, 23-36. DOI: 10.1016/01683659(87)90034-4.

18. Costa, P.; Sousa Lobo, J. M. Modeling and comparison of dissolution profiles. Eur. J. Pharm. Sci. 2001, 13,123-133. DOI: 10.1016/S0928-0987(01)00095-1.

19. Sarode, A. L.; Sandhu, H.; Shah, N.; Malick, W.; Zia, H. Hot melt extrusion for amorphous solid dispersions: temperature and moisture activated drug-polymer interactions for enhanced stability. Mol. Pharm. 2013, 10, 3665-3675. DOI: 10.1021/ mp400165b.

20. Vasvári, G.; Kalmár, J.; Veres, P.; Vecsernyés, M.; Bácskay, I.; Fehér, P.; Ujhelyi, Z.; Haimhoffer, Á.; Rusznyák, Á.; Fenyvesi, F.; Váradi, J. Matrix systems for oral drug delivery: Formulations and drug release. Drug Discov. Today Technol. 2018, 27, 71-80. DOI: 10.1016/j.ddtec.2018.06.009.

21. Nerurkar, J.; Jun, H. W.; Price, J. C.; Park, M. O. Controlledrelease matrix tablets of ibuprofen using cellulose ethers and 
carrageenans: effect of formulation factors on dissolution rates. Eur. J. Pharm. Biopharm. 2005, 61, 56-68. DOI: 10.1016/j. ejpb.2005.03.003.

22. Maderuelo, C.; Zarzuelo, A.; Lanao, J. M. Critical factors in the release of drugs from sustained release hydrophilic matrices. J. Control. Release. 2011, 154, 2-19. DOI: 10.1016/j. jconrel.2011.04.002.
23. Markl, D.; Zeitler, J. A. A review of disintegration mechanisms and measurement techniques. Pharm. Res. 2017, 34, 890-917. DOI: 10.1007/s11095-017-2129-z.

24. Agrawal, A. M.; Dudhedia, M. S.; Zimny, E. Hot melt extrusion: development of an amorphous solid dispersion for an insoluble drug from mini-scale to clinical scale. AAPS PharmSciTech. 2016, 17, 133-147. DOI: 10.1208/s12249-015-0425-7. 